\title{
TBK1 Participates in Glutaminolysis by Mediating the Phosphorylation of RIPK3 to Promote Endotoxin Tolerance
}

\section{Lehan Pan}

The Second Affiliated Hospital of Chongqing Medical University

\section{Lian Yang}

The Second Affiliated Hospital of Chongqing Medical University

\section{Zhujun Yi}

The Second Affiliated Hospital of Chongqing Medical University

\section{Wenfeng Zhang}

The Second Affiliated Hospital of Chongqing Medical University

Jianping Gong ( $\mathbf{} 300381 @$ @ospital.cqmu.edu.cn )

The Second Affiliated Hospital of Chongqing Medical University https://orcid.org/0000-0002-59267944

\section{Research Article}

Keywords: TBK1, Endotoxin tolerance, Macrophage, RIPK3, GLUD1

Posted Date: February 15th, 2022

DOI: https://doi.org/10.21203/rs.3.rs-1236153/v1

License: (c) (i) This work is licensed under a Creative Commons Attribution 4.0 International License. Read Full License 


\section{Abstract}

Background: TBK1 (TRAF-associated NF-KB activator (TANK)-binding kinase 1), a nonclassical IKB kinase (IKK), and its effect on inflammation have not been entirely clarified. This study aimed to determine how TBK1 participates in the catabolism of glutamine by mediating the phosphorylation of receptorinteracting protein kinase 3 (RIPK3) and promoting macrophage endotoxin tolerance (ET).

Methods: Lipopolysaccharide (LPS)-induced ET mice models were used for in vivo studies. Hematoxylin and eosin staining, immunofluorescence staining and immunohistochemical staining protocols were used to analyze histological changes in the liver, and the related key pathway proteins were analyzed by western blotting. Kupffer cells (KCs) and bone marrow-derived macrophages (BMDMs) isolated from C57BL/ 6 mice were used to establish ET models. ELISA was used to detect the levels of necrosis factor-a (TNF-a), interleukin-10 (IL-10) and a-ketoglutarate ( $\mathrm{a}-\mathrm{KG}$ ). The differentiation of M1/M2 macrophages was assessed by flow cytometry for assessing the cell-surface markers, CD86 and F4/80. The related key proteins were analyzed by western blotting and immunofluorescence staining.

Results: The phosphorylation of TBK1 was higher in the ET group than in the NET group in vitro and in vivo. As TBK1 was overexpressedd by shRNA, the phosphorylation level of TBK1 was increased, and the protective effect of ET was also strengthened. The TBK1 protein directly interacts with the RIPK3 protein and mediates the phosphorylation of RIPK3 in macrophages. According to our co-IP results, the glutamate dehydrogenase 1 (GLUD1) and RIPK3 proteins interact in ET models. In addition, activated RIPK3 can directly bind to GLUD1, thereby improving its catalytic activity and increasing the production of a-ketoglutarate ( $\mathrm{a}-\mathrm{KG}$ ) in macrophages. a-KG generated from glutaminolysis promotes $\mathrm{M} 2$ activation and restricts M1 polarization to induce the macrophage tolerance of LPS. These findings reveal a novel mechanism for the metabolic control of inflammation and for the induction of ET by modulating glutamine metabolism.

Conclusion: As a result of TBK1 regulating the phosphorylation level of RIPK3, overexpressed TBK1 could enhance the tolerance of macrophages to endotoxin through glutaminolysis, which unveils the potential for therapeutic targeting of the metabolic status of macrophages.

\section{Background}

Inflammation is the general protective host response to stimulation by invading pathogens or endogenous signals, such as damaged cells, the clearance of necrotic cells, and tissue repair (Netea et al. 2017). Toll-like receptor 4 (TLR4) is the major pattern recognition receptor (PRR) involved in the detection of gram-negative bacteria and their associated endotoxins (Biswas et al. 2007; Petrasek et al. 2013). However, uncontrolled inflammation leads to extensive tissue damage and manifestations of pathological states such as sepsis (Venet and Monneret 2018). Pathophysiological adaptations to regulate overexuberant inflammation serve as an important mechanism for host protection against endotoxin shock. One of these protective mechanisms is endotoxin tolerance (ET) (Biswas and Lopez- 
Collazo 2009). Long-term exposure to lipopolysaccharide (LPS) or injection of sublethal doses of LPS in animals can induce tolerance of endotoxin that reprograms the inflammatory response, resulting in cells or organisms entering into a transient unresponsive state where they are unable to respond to further challenges with endotoxin (Biswas and Lopez-Collazo 2009; Liu et al. 2019). In several studies that have addressed the molecular basis of ET, LPS unresponsiveness has been associated with decreased expression of nuclear factor-KB (NF-KB) (Del et al. 2009).

TANK (TRAF-associated NF-KB activator) binding kinase 1 (TBK1), which is ubiquitously expressed in all tissues, is a noncanonical IKB kinase (IKK) (Shin and Choi 2019; Pomerantz and Baltimore 1999). Although TBK1 has a domain composition similar to that of canonical IKKs, it appears that TBK1 does not generally target NF-KB signaling and that the role of TBK1 in NF-KB activation is highly dependent on cellular and signal-induced contexts (Shin and Choi 2019; Perry et al. 2004; Chau et al. 2008). Studies have shown that TBK1 can suppress inflammation by phosphorylating and inducing the degradation of the IKK kinase NF-KB-inducing kinase (NIK), thus attenuating NF-KB activity to reduce inflammation (Zhao et al. 2018; Jin et al. 2012). In addition, TBK1 deficiency in cells promotes the recruitment of proinflammatory macrophages, which release inflammatory cytokines such as tumor necrosis factor-a (TNF-a) and induce NF-KB activation in mice (Zhao et al. 2018). Therefore, we speculate that TBK1 might be involved in the regulation of macrophage $\mathrm{ET}$.

Receptor-interacting protein kinase 3 (RIPK3) can induce necrotic death termed necroptosis when caspase activities are compromised by either viral or chemical inhibitors (Sun et al. 2012; Davies et al. 2017). Several reports focusing on macrophages suggest that RIPK3 regulates the production of proinflammatory cytokines in particular settings and modulates NF-KB activation (Kang et al. 2013; Wong et al. 2014; Newton 2015). Moreover, RIPK3 overexpression can promote the activation of the immunoresponsive gene 1 (IRG1)-itaconate pathway to restrict viral replication in vitro and promote survival following intracranial virus challenge in vivo (Zhao et al. 2018). Together, RIPK3 potentially initiates many cellular responses, which exert multifaceted control over cell death and inflammatory signaling pathways (Sun et al. 1999; Zhang et al. 2009; Speir and Lawlor 2021).

According to reports, after TNF-a stimulates cells, activated RIPK3 can directly bind to glutamate dehydrogenase 1 (GLUD1), which is known as a critical enzyme for catalyzing glutamine decomposition, thus improving its catalytic activity and increasing the production of a-ketoglutarate ( $a-K G)$ in macrophages (Mastorodemos et al. 2009; He et al. 2020). After inhibiting the activation of RIPK3 induced by LPS, it can directly reduce the catalytic activity of GLUD1 (Nelson et al. 2018).

Macrophages have remarkable plasticity that allows them to efficiently respond to environmental signals and change their phenotype, and their physiology can be markedly altered by both innate and adaptive immune responses (Mosser and Edwards 2008). The phenotypic remodeling of macrophages must also be accompanied by a change in cell energy metabolism during inflammation induced by endotoxin. Growing evidence suggests that macrophages can limit the invasion of bacteria and other microorganisms by increasing the catabolism of glutamine (Davies et al. 2017; Cruzat et al. 2018). More 
importantly, the glutamine metabolic pathway is also involved in the specific procedures required for macrophage polarization (Davies et al. 2017; Nelson et al. 2018; Liu et al. 2017). It has been reported that a-KG generated from glutaminolysis can promote M2 activation via Jmjd3-dependent metabolic and epigenetic reprogramming (Liu et al. 2017). In contrast, a-KG impairs proinflammatory responses in M1 macrophages by suppressing the NF-KB pathway. While glutaminolysis restricts M1 polarization, a-KG produced from glutaminolysis during LPS stimulation has a crucial role in promoting LPS-induced ET in macrophages (Cruzat et al. 2018; Liu et al. 2017).

Overall, these studies have revealed the roles of TBK1 and RIPK3 in endotoxin-induced inflammation and suggested that RIPK3 and GLUD1 play essential roles in modulating the polarization of macrophages by regulating the production of a-KG. We report here that the TBK1-RIPK3 pathway enhances the catalytic activity of GLUD1 to accelerate the catabolism of glutamine to produce more $\mathrm{a}-\mathrm{KG}$, thereby participating in the regulation of phenotypic remodeling and inducing macrophage ET. These results highlight the mechanisms controlled by glutaminolysis to fine-tune macrophage activities and suggest that modulation of glutamine metabolism would be an attractive strategy for harnessing macrophagemediated immune response.

\section{Methods And Materials}

\section{Cell isolation}

RAW264.7 cells were obtained from the Laboratory of the Second Affiliated Hospital of Chongqing Medical University (Chongqing, China) and cultured in DMEM (Gibco Life Technologies) with 10\% FBS (PAN-Biotechnology) and $1 \%$ penicillin-streptomycin (Beyotime Biotechnology) at $37^{\circ} \mathrm{C}, 95 \%$ humidity, and $5 \% \mathrm{CO}_{2}$. The isolation methods of bone marrow-derived macrophages (BMDMs) and Kupffer cells (KCs) from C57BL/6 mice were performed according to the steps in the approach proposed by $\mathrm{Li}(\mathrm{Li}$ et al. 2014). The cells were divided into three groups: the control group (CON) (treated with PBS), the endotoxin tolerance group (ET) (treated with $10 \mathrm{ng} / \mathrm{mL}$ LPS for $24 \mathrm{~h}$, then $1000 \mathrm{ng} / \mathrm{mL}$ LPS for $12 \mathrm{~h}$ ) and nonendotoxin tolerance group (NET) (treated with $1000 \mathrm{ng} / \mathrm{mL}$ LPS for $12 \mathrm{~h}$ ).

\section{Reagents}

We used antibodies against TBK1 (Abcam, ab40676, 1:1000), phospho-TBK1/NAK (Ser172) (Cell Signaling, \#5483, 1:1000), RIPK3 (Cell Signaling, \#95702, 1:1000), phospho-RIPK3 (Cell Signaling, \#91702, 1:1000), NF-kB (Proteintech, 10745-1-AP, 1:1000), phospho-NF-KB (Cell Signaling, \#3033, 1:1000), GLUD1 (Abcam, ab168352, 1:1000), TNF-a (Abcam, ab183218, 1:1000), iNOS (Abcam, ab178945, 1:1000), GAPDH (Servicebio, GB12002, 1:1000). CD86 (Biolegend,105007), CD163 (Biolegend, 333606), and F4/80 (Biolegend, 123109).

HRP-conjugated anti-rabbit and anti-mouse secondary antibodies were obtained from Boster Biological Technology (Wuhan, China). LPS was purchased from Sigma (L2880). Neutral liposomes and control 
liposomes (F70101C-NC-2) were purchased from FormuMax. M-CSF (576402) was obtained from Biolegend.

\section{siRNA and lentivirus transduction}

All small interfering RNAs (siRNAs) (TBK1, RIPK3) and control siRNAs were prepared by GenePharma (Shanghai) and all short hairpin RNAs (shRNAs) (shRNA-PGC-1a, shRNA-mtTFA and control shRNAs) were prepared by GeneChem (Shanghai). siRNA and lentiviral transduction were performed according to the manufacturer's instructions. For siRNA transduction, $2 \times 10^{5}$ RAW264.7 cells (MOI=20) were cultured in $2 \mathrm{ml}$ medium with $200 \mathrm{pmol}$ siRNA and $5 \mu \mathrm{l}$ siRNA Mate (GenePharma), and the culture medium was changed after $6 \mathrm{~h}$. For lentivirus transduction, $1 \times 10^{5}$ RAW264.7 cells (MOI=20) in $1 \mathrm{ml}$ medium with 10 $\mathrm{mg} / \mathrm{ml}$ of polybrene (GeneChem) were incubated with $2 \mu$ lentivirus. After $72 \mathrm{~h}$ of culture, western blotting was used to analyze the transduction efficiency.

\section{Animals and protocols}

Male C57BL/ 6 mice (8-10 weeks old, 22 $\pm 2 \mathrm{~g}$ ) were used for all experiments (Experiments Animal Center of Chongqing Medical University, Chongqing, China). Animals were fed standard rodent chow in a temperature-controlled environment with $50 \%$ humidity and $12 \mathrm{~h}$ light/dark cycles in cages of five mice. To ensure the accuracy of the research, the mice were divided randomly into the following groups: normal saline group (CON) (mice were treated with intraperitoneal injections of sterile normal saline), endotoxin tolerance group (ET) (mice were pretreated with intraperitoneal injections of $50 \mu \mathrm{g} / \mathrm{kg}, 250 \mu \mathrm{g} / \mathrm{kg}$ and 500 $\mu \mathrm{g} / \mathrm{kg}$ LPS for three consecutive days, and twelve hours after the last injection, mice were treated with intraperitoneal injections of $20 \mathrm{mg} / \mathrm{kg}$ LPS), nonendotoxin tolerance group (NET) (mice were intraperitoneally injected with $20 \mathrm{mg} / \mathrm{kg}$ LPS), control liposome group (mice were treated with an intraperitoneal injection of control liposomes) and neutral liposome group (mice were treated with an intraperitoneal injection of neutral liposomes).

Survival and weight changes were monitored once per $24 \mathrm{~h}$ period for 6 days, and then liver and blood samples were collected.

\section{ELISA}

The levels of TNF-a, interleukin-10 (IL-10) and a-KG in mouse serum and cell supernatants were determined by using ELISA kits from Jbswbio Biological Technology according to the manufacturer's instructions.

\section{Flow cytometry analysis}

To determine the macrophage differentiation phenotypes, BMDMs were isolated from C57BL/ 6 mice and NET and/or ET models were established as previously reported. Then, adherent cells were collected for flow cytometry analysis of F4/80 and CD86. RAW264.7 cells were separately stained with a monoclonal antibody specific for PE-CD86. Both cell cultures were then analyzed using a FACSVerse flow cytometer (BD Biosciences, San Jose, CA, USA). 


\section{Western blot}

After washing with PBS for 3 times, the cells were lysed in a lysis solution containing a protease inhibitor, phosphatase inhibitors and PMSF (KeyGen, China) and then sonicated on ice. After centrifugation (15 min, $12000 \times \mathrm{g}, 4^{\circ} \mathrm{C}$ ), the supernatant was mixed with SDS-PAGE loading buffer and heated to $100^{\circ} \mathrm{C}$ for $10 \mathrm{~min}$. The concentration of protein was determined by an Enhanced BCA Protein Assay Kit (Beyotime, China). The proteins were separated by SDS-PAGE (Bio-Rad, Hercules, CA, USA) and electrotransferred to PVDF membranes (Millipore, Billerica, MA, USA). The membranes were blocked in 5\% BSA (Solarbio, Shanghai, China) for $1 \mathrm{~h}$ at $37^{\circ} \mathrm{C}$. The corresponding primary antibodies were incubated with the membranes used overnight at $4^{\circ} \mathrm{C}$. After being washed, the membranes were incubated with an HRPconjugated anti-rabbit or anti-mouse secondary antibody $(1: 5000)$ for $1 \mathrm{~h}$ at $37^{\circ} \mathrm{C}$. Finally, the membranes were washed and stained with the Ultra High Sensitivity ECL Kit (MCE, China). The stained target proteins were detected by the Quantity One gel scanning system (Bio-Rad, Hercules, CA, USA).

\section{GLUD1 enzyme activity assay}

The enzyme activities of GLUD1 in mouse livers and macrophages were determined using a micro GLUD1 assay kit (Solarbio, BC1465) according to the manufacturer's instructions.

\section{Immunofluorescence staining}

Cells were fixed by immersion in methanol for 10 min and permeabilized with $0.3 \%$ Triton X-100 at room temperature for $10 \mathrm{~min}$. After blocking with $5 \% \mathrm{BSA}$ at room temperature for $60 \mathrm{~min}$, the cells were incubated at $4^{\circ} \mathrm{C}$ overnight with primary antibodies. The next day, after a brief wash with PBS, the cells were incubated with the secondary antibody (1:500) at room temperature in the dark for $1 \mathrm{~h}$. Finally, the cells were incubated with DAPI in the dark at room temperature for $10 \mathrm{~min}$. After sealing with a fluorescent quenching agent, stained cells were observed by laser scanning confocal microscopy (LSCM).

\section{Histological analysis}

Liver tissues were fixed in $10 \%$ neutral formalin, dehydrated, embedded in paraffin, and cut into $5-\mu \mathrm{m}$ thick sections. The paraffin sections were stained with hematoxylin and eosin (H\&E). The sections were stained using commercial kits (ZSGB-BIO, China) for immunohistochemical staining following the manufacturers' instructions. After dewaxing, hydration and antigen repair of the tissue sections, the tissue sections were incubated with an antibody at $4^{\circ} \mathrm{C}$ overnight. The corresponding IgG polymer, DAB and hematoxylin were used. After dehydration and sealing, images were observed and collected by microscopy.

\section{Liver function analysis}

The alanine aminotransferase (ALT) and aspartate aminotransferase (AST) levels in serum were measured using commercial kits (Solarbio, BC1550 and BC1560).

\section{Coimmunoprecipitation}


Cells were treated with cell lysis buffer (Beyotime, China) to obtain cell lysates and retain the input material. Agarose beads (Protein A Agarose, Beyotime, China) were incubated with an antibody at $4^{\circ} \mathrm{C}$ for $2 \mathrm{~h}$ to ensure that the agarose beads bound to the antibody. Agarose beads bound to the antibody were added to the cell lysate and shaken at $4^{\circ} \mathrm{C}$ overnight. The sample protein was obtained by repeated elution of nonspecific binding proteins. The sample was mixed with SDS-PAGE loading buffer and heated to $100^{\circ} \mathrm{C}$ for $10 \mathrm{~min}$. The results were detected by western blot. IPKine ${ }^{\mathrm{TM}} \mathrm{HRP}$, Mouse Anti-Rabbit IgG LCS (Abbkine, China) was used as the secondary antibody.

\section{Statistical analysis}

Statistical analysis of the data was performed using Prism software (GraphPad Prism version 8.0). The standard error of the mean was calculated from the average of at least 3 independent samples under a given treatment condition. Statistically significant differences between two groups were determined by a two-tailed Student's $t$ test. One-way ANOVA was used for multiple group comparisons. And a p value of

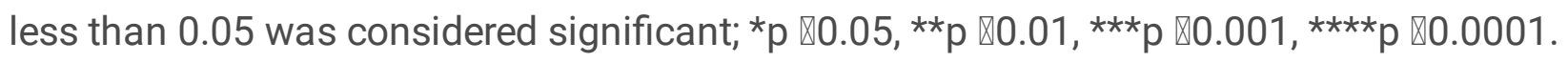

\section{Results}

\section{A low dose of LPS induces ET in vitro and in vivo}

LPS has the ability to incite a vigorous inflammatory response and result in all the physiological manifestations of septic shock (Su 2002). However, after exposure to low concentrations of LPS, cells or organisms can enter into a transient unresponsive state and are unable to respond to further challenges with LPS (Seeley and Ghosh 2017). This phenomenon is ET, one of the important mechanisms for host protection against endotoxin shock.

To explore the mechanism of ET, we stimulated C57BL/6 mice with low-dose LPS and established ET models in vivo and $\mathrm{KCs}$ in vitro. $\mathrm{KCs}$ isolated from $\mathrm{C} 57 \mathrm{BL} / 6$ mice were randomly divided into three groups: the control (CON) group, the nonendotoxin tolerance (NET) group and the endotoxin tolerance (ET) group (NET: $1000 \mathrm{ng} / \mathrm{ml}$; ET: $10 \mathrm{ng} / \mathrm{ml}+1000 \mathrm{ng} / \mathrm{ml}$ ). The ET and NET groups were cultured with complete medium containing $1000 \mathrm{ng} / \mathrm{ml}$ LPS for $12 \mathrm{~h}$, but before that, the ET group was pretreated with $10 \mathrm{ng} / \mathrm{mL}$ LPS for $24 \mathrm{~h}$. The ET group had lower expression of proinflammatory cytokines including TNF$a$ and iNOS (Fig. 1A). Additionally, the level of TNF-a in the cell supernatants of the NET group was significantly increased compared to that in the ET group, while the level of IL-10 in the NET group was lower than that in the ET group (Fig. 1B).

To study the effect of ET in vivo, mice in the ET group were pretreated with LPS (intraperitoneal injections of $50 \mu \mathrm{g} / \mathrm{kg}, 250 \mu \mathrm{g} / \mathrm{kg}, 500 \mu \mathrm{g} / \mathrm{kg}$ LPS for three consecutive days) and then the two groups (NET and ET) were restimulated with LPS ( $20 \mathrm{mg} / \mathrm{kg}$, intraperitoneally) for $24 \mathrm{~h}$. All of the mice were observed for 6 days, and we found that compared to NET group, mice in ET group had significantly improved survival (Fig. 1C). No mice died during the observation period in the saline-treated group (CON), which was not 
reflected in the survival curve. The liver sections from each group showed less tissue damage and inflammatory cell infiltration in the ET group (Fig. 1D), and the ET group had reduced levels of

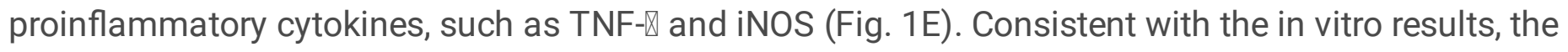
serum levels of TNF-a in the NET group were higher than that in the ET group, and the levels of IL-10 in the NET group were decreased compared to that in the ET group (Fig. 1F). In addition, the serum AST and ALT levels showed that the ET group experienced lower inflammatory activation and tissue damage (Fig. 1G). The above results demonstrated that the ET model was successfully established in vitro and in vivo.

\section{TBK1 can mediate inflammatory regulation in ET}

It was reported that promoting TBK1 phosphorylation can regulate the host innate immune response during infection (Chen 2021). To determine whether TBK1 was involved in ET, mice were treated as previously described to establish ET and NET models in vivo. In both the NET and ET groups, the phosphorylation of TBK1 increased; however, the phosphorylation of TBK1 was higher in the ET group than in the NET group. In addition, the ET group had fewer proinflammatory cytokines, such as TNF- $\triangle$ and iNOS (Fig. 2A). A higher level of TBK1 phosphorylation was also found in the ET group according to immunofluorescence staining (Fig. 2B). To clarify the relationship between TBK1 and ET in vitro, KCs isolated from normal C57BL/ 6 mice were treated as previously described. While total TBK1 was not affected in either the NET or ET groups (Fig. 2C), the phosphorylation of TBK1 in the ET group was increased to a greater extent (Fig. 2C-D), which suggested that TBK1 might be related to ET in vivo and in vitro.

To explore the role of TBK1 in ET, TBK1 expression was knocked down in RAW264.7 cells with siRNA (TBK1-siRNA). The TBK1-siRNA+ET group exhibited downregulated phosphorylation of TBK1 compared with the ET group, and the expression of proinflammatory cytokines, including TNF- $\$ and iNOS, was increased, which was the reverse of the previous results (Fig. 2E). As TBK1 was knocked down by siRNA, the phosphorylation level of TBK1 was decreased, and the protective effect of ET was also weakened. Correspondingly, the TBK1 gene was overexpressed in RAW264.7 cells by shRNA-TBK1 lentiviral transduction (LV-TBK1-OE). As predicted, the protein level of TBK1 increased significantly after lentiviral transduction for $72 \mathrm{~h}$. The LV-TBK1-OE+ET group had the lower expression of proinflammatory cytokines (including TNF- $\triangle$ and iNOS) and higher phosphorylation of TBK1 (Fig. 2F). The serum level of TNF-a in the TBK1-siRNA+ET group was significantly increased compared to the LV-TBK1-OE+ET group, while the serum level of IL-10 in the TBK1-siRNA+ET group was lower than that in the LV-TBK1-OE+ET group (Fig. 2G). These results revealed that the ET was partly dependent upon TBK1 in mediating the reduction of inflammation, and the phosphorylation level of TBK1 was closely related to the level of inflammation during ET: when TBK1 was knocked down and the phosphorylation level decreased, inflammation was aggravated and the secretion of inflammatory factors increased, while when TBK1 was overexpressed and the phosphorylation level increased, the inflammation was alleviated. The above results indicated that TBK1 could mediate inflammatory regulation in ET through protein phosphorylation. 


\section{TBK1 can regulate the phosphorylation of RIPK3}

Recent studies focusing on macrophages indicated that RIPK3 can regulate the production of proinflammatory cytokines in particular settings (Kang et al. 2013; Wong et al. 2014; Newton 2015). RIPK3 may be involved in regulating cell death and inflammatory signaling pathways (Sun et al. 1999; Zhang et al. 2009), therefore, we tried to explore whether RIPK3 played a role in ET. After stimulation with LPS, KCs in the ET group showed high expression of phosphorylated RIPK3 compared to KCs in the NET group (Fig. 3A). Immunofluorescence staining also confirmed that the phosphorylation level of RIPK3 was increased in the ET group (Fig. 3B). These results revealed that RIPK3 might be involved in ET. In addition, RIPK3 was found to interact with TBK1 by coimmunoprecipitation (co-IP) experiments established in KCs with coexpressed proteins in the ET group (Fig. 3C), which indicated that TBK1 and RIPK3 can interact during macrophage tolerance to LPS.

To further explore the relationship between TBK1 and RIPK3 in ET in macrophages, we first knocked down the expression of TBK1 in RAW264.7 cells to observe whether the change in TBK1 expression would affect the expression of RIPK3. As the phosphorylation level of TBK1 decreased, the level of phosphorylated RIPK3 was decreased (Fig. 3D). As predicted, the phosphorylation levels of TBK1 and RIPK3 were both upregulated after increasing the expression of TBK1 by shRNA-TBK1 lentiviral transduction in the LV-TBK1-OE+ET group (Fig. 3E). These results revealed that TBK1 could affect the phosphorylation level of RIPK3 in ET, but the functional significance of the RIPK3 phosphorylation event and downstream substrates have not been fully clarified (Sun et al. 2012; Declercq et al. 2009).

\section{Increasing the enzymatic activity of GLUD1 can induce ET in macrophages}

GLUD1 is known as a mitochondrial matrix enzyme that converts glutamine to a-KG (Zhang et al. 2009; He et al. 2020; Goossens et al. 1996), and growing evidence has shown that glutamine can transfer into mitochondria to function as an energy substrate (Zhang et al. 2009). The protein expression of GLUD1 and phosphorylated RIPK3 was higher in the ET group than in the NET group (Fig. 4A).

Immunofluorescence staining showed that the expression of GLUD1 increased in the ET group (Fig. 4B). Furthermore, the enzyme activities of GLUD1, both in the liver and in BMDMs (the models were established as previously described), were significantly increased in the ET group compared with the NET group (Fig. 4C), while immunohistochemistry was also confirmed that the ET group had a higher expression of GLUD1 than the NET group (Fig. 4D). These results suggested that GLUD1 expression was indeed increased in ET, but how GLUD1 promotes LPS tolerance remains unclarified.

Macrophages represent with distinct phenotypes with different functions according to the tissue microenvironment and different stimuli (Wynn et al. 2013; Murray and Wynn 2011). Liu reported that a-KG 
generated from glutaminolysis could promote M2 activation via Jmjd3-dependent metabolic and epigenetic reprogramming, while glutaminolysis restricted M1 polarization (Liu et al. 2017). Therefore, we carried out ELISAs to detect the contents of a-KG in the different groups. The levels of a-KG in serum and cell supernatants in the ET group were than those in the NET group (Fig. 4E). We then checked the ratios of M1-type and M2-type macrophages among BMDMs. As predicted, the ratio of M1-type macrophages in the ET group was significantly decreased (Fig. 4F). The expression of CD86 was increased in the NET group (Fig. 4G). As the enzyme activity of GLUD1 increased, the content of a-KG also increased restricting M1 polarization and promoting macrophage tolerance to LPS. Increasing the enzyme activity of GLUD1 could be an effective measure to induce ET in macrophages.

\section{The regulatory effect of GLUD1 on ET was reversed after eliminating macrophages in mice}

Macrophages are critical to maintain the tissue homeostasis in innate and adaptive immune responses (Wynn et al. 2013). To determine whether the elimination of macrophages would have an effect on the induction of ET by GLUD1, we depleted macrophages in mice with clodronate (neutral) liposomes. Male C56BL/6J mice (8-10 weeks old, $22 \pm 2 \mathrm{~g}$ ) were randomly divided into two groups: the control liposome group (injected peritoneally with $200 \mu \mathrm{l}$ control liposomes) and the neutral liposome group (injected peritoneally with $200 \mu \mathrm{l}$ neutral liposomes). The mice in the two groups were treated as previously described to establish ET in vivo. The control liposome group was divided into three groups: the control liposome control (C-CON) group, the control liposome NET (C-NET) group and the control liposome ET (CET) group, while the neutral liposome group was also divided into three groups: the neutral liposome CON (N-CON) group, the neutral liposome NET (N-NET) group and the neutral liposome ET (N-ET) group (Fig. 5A).

The KCs in the livers of mice in the control liposome group remained essentially unchanged, while the $\mathrm{KCs}$ in the neutral liposome group were basically eliminated (Fig. 5B). All of the mice were observed for 6 days, and we found that compared to the C-NET group, mice in the C-ET group had significantly improved survival. However, compared to the N-NET group, the survival of mice in the N-ET group was significantly decreased which was the reverse of the above results. Compared with the C-ET group, the percent survival of mice in the N-ET group was reduced by approximately $93 \%$. Moreover, all mice in the N-ET group died before the sixth day of observation (Fig. 5C). H\&E staining of the liver sections from each group showed that there was less tissue damage and inflammatory cell infiltration in the C-ET group than in the C-NET group, while there were no significant differences in tissue injury and inflammatory cell infiltration between the N-NET group and the N-ET group (Fig. 5D). Consistent with the H\&E results: the serum levels of TNF-a and IL-10 were not significantly different between the N-NET and N-ET groups (Fig. 5E). Furthermore, the expression of GLUD1 was lower in the N-ET group than that in the C-ET group (Fig. 5F). The enzyme activity of GLUD1 was decreased in the N-ET group compared with the C-ET group, resulting in a decrease in the content of a-KG in the liver (Fig. 5G-H). The inflammatory response in the neutral 
liposome group was still severe because the protective mechanism of ET was not induced, even if mice were stimulated with low dose LPS in advance. Taken together, these results indirectly proved that GLUD1 participated in ET by regulating macrophages and that the regulatory effect of GLUD1 on ET was reversed after eliminating the macrophages in mice.

\section{RIPK3 induces ET by promoting the enzyme activity of GLUD1}

The interaction of endogenous RIPK3 with GLUD1 increases after TNF-a stimulation, and in cells that have sufficient RIPK3 expression, the gateway to glutamine use is readily opened (Zhang et al. 2009). In addition, it could directly reduce the catalytic activity of GLUD1 after inhibiting the activation of RIPK3 induced by LPS (Nelson et al. 2018). Based on the facts of previous studies, we tried to explore the roles of RIPK3 and GLUD1 in ET. According to our co-IP results, the GLUD1 and RIPK3 proteins interact in ET models in vivo and in vitro (Fig. 6A-B).

To further determine the regulatory effect of RIPK3 on GLUD1, we first knocked down RIPK3 in RAW264.7 cells using siRNA and established ET and NET models (RIPK3-siRNA). The protein levels of RIPK3 and phosphorylated RIPK3 decreased significantly in the RIPK3-siRNA+ET group. As shown in Fig. 6C, with the decrease in RIPK3, the expression of GLUD1 in the RIPK3-siRNA+ET group was significantly lower

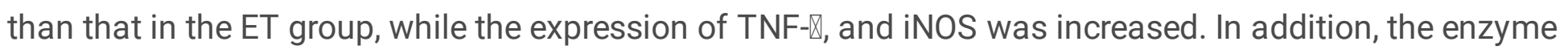
activity of GLUD1 in the RIPK3-siRNA+ET group was much lower than that in the ET group (Fig. 6D). As the level of $a-K G$ was decreased in the RIPK3-siRNA+ET group compared with the ET group (Fig. 6E), the ratio of M1 macrophages in the RIPK3-siRNA+ET group was increased (Fig. 6F). After knocking down the expression of RIPK3, the enzyme activity of GLUD1 decreased, and the production of a-KG decreased, resulting in a reduction in macrophage tolerance to LPS.

Correspondingly, when the RIPK3 gene was overexpressed in RAW264.7 cells by shRNA-RIPK3 lentiviral transduction (LV-RIPK3-OE), GLUD1 expression increased, while the enzyme activity of GLUD1 also increased (Fig. 6G-H), which led to a higher level of a-KG in the LV-RIPK3-OE+ET group (Fig. 6I). These results indicated that increasing RIPK3 can promote the enzyme activity of GLUD1 to increase the level of a-KG. The expression of iNOS and TNF- $\triangle$ in the LV-RIPK3-OE+ET group was significantly decreased after increasing the expression of RIPK3, which showed that overexpression of RIPK3 can promote the enzyme activity of GLUD1, thus strengthening the effect of a-KG on promoting ET and alleviating the inflammatory reaction (Fig. 6G). Taken together, RIPK3 can mediate the inflammatory response induced by LPS and enhance ET through glutaminolysis.

\section{TBK1 participates in glutaminolysis by mediating the phosphorylation of RIPK3 to promote ET}


TBK1 is required in ET both in vivo and in vitro; however, the specific mechanism of how TBK1 participate in ET is still unclear. As TBK1 promoted the phosphorylation of RIPK3, we next sought to determine whether TBK1 participated in the glutamine metabolic pathway to promote ET. We knocked down the expression of TBK1 by using siRNA and found that the expression of GLUD1 in the TBK1-siRNA+ET group was lower than that in the ET group (Fig. 7A), as well as the enzyme activity of GLUD1 (Fig. 7B). In addition, the level of $a-K G$ was also decreased in the TBK1-siRNA+ET group compared with the ET group (Fig. 7C). Flow cytometry showed that the ratio of M1 macrophages was lower in the TBK1-siRNA+ET group than in the ET group (Fig. 7D). Taken together, these findings demonstrated that the decrease in TBK1 expression could reduce the expression of phosphorylated RIPK3 and then affect the enzyme activity of GLUD1, resulting in a decrease in the production of a-KG. Finally, shRNA-TBK1-OE lentiviral particles were used to increase TBK1 expression in RAW264.7 cells. Compared with the ET group, overexpression of TBK1 could successfully increased the expression of GLUD1 (Fig. 7E). Additionally, the enzyme activity of GLUD1 increased, and the level of a-KG increased in the LV-TBK1-OE+ET group compared with the ET group (Fig. 7F-G). All of these findings indicated that the expression of TBK1 can mediate the phosphorylation of RIPK3, thereby inducing GLUD1 to decompose glutamine to produce aKG. These results suggested that the modulation of glutamine metabolism, which plays a vital role in macrophage ET, would be an attractive strategy for harnessing macrophage-mediated immune responses.

\section{Discussion}

ET serves as a protective mechanism to reduce the overproduction of proinflammatory cytokines in response to infection. Defects in the establishment of ET lead to a higher incidence of septic shock and mortality in individuals with infection. However, individuals with ET become immunocompromised (Nathan and Ding 2010). Therefore, understanding the mechanisms controlling ET is important to the design of interventions to fine tune immune responses. Here, we present a novel function by which TBK1 participates in glutaminolysis by mediating the phosphorylation of RIPK3 to promote macrophage tolerance to endotoxin. The TBK1 protein directly interacts with the RIPK3 protein and mediates the phosphorylation of RIPK3 in macrophages. Then, activated RIPK3 directly improves the enzyme activity of GLUD1, which increases the production of a-KG to restrict M1 polarization, resulting in the promotion of macrophage tolerance to LPS (Fig. 8).

Macrophages are one of the major cell types involved in ET, and LPS, a TLR4 ligand, is most commonly used to induce ET in vivo and in vitro (Biswas and Lopez-Collazo 2009; Ho et al. 2012). TBK1, one of the noncanonical IKKs that has an undefined role in NF-KB activation, is phosphorylated by proinflammatory cytokines and ligands that bind to TLR4 (Pomerantz and Baltimore 1999; Perry et al. 2004). Interestingly, TBK1 can inhibit inflammation by phosphorylating and inducing the degradation of NIK, thereby reducing the activity of NF-KB (Zhao et al. 2018; Jin et al. 2012). Moreover, TBK1 deficiency in cells increases the recruitment of proinflammatory macrophages, which release inflammatory cytokines such as TNF-a 
(Zhao et al. 2018). Therefore, we speculate that TBK1 is involved in the polarization of macrophage and plays a role in macrophage ET. In our study, we found that the phosphorylation of TBK1 in KCs in the ET group was higher than that in KCs in the NET group, and the effect of inducing ET was weakened after the expression of TBK1 was inhibited by siRNA. When the phosphorylation level of TBK1 increased, the tolerance of macrophages to endotoxin was also strengthened. Consequently, TBK1 is a target molecule that regulates the tolerance of macrophages to endotoxin.

RIPK3 can mediate a form of cell death called necroptosis when apoptosis is blocked (Sun et al. 2012; Newton 2015; Declercq et al. 2009; Dowling et al. 2019). A common assumption is that RIPK3 promotes inflammation because necroptosis releases intracellular components that stimulate innate immune cells (Newton 2015; Pasparakis and Vandenabeele 2015). However, RIPK3 has additional functions beyond inducing cell death. Several recent reports focusing on macrophages suggest that RIPK3 regulates the production of proinflammatory cytokines in particular settings (Kang et al. 2013; Wong et al. 2014; Newton 2015). In addition, the modulation of NF-kB activation by RIPK3 was observed (Moriwaki and Chan 2013). Therefore, the biological function of RIPK3 in inflammation is largely unknown (Newton 2015). In our study, the TBK1 protein directly interacted with the RIPK3 protein in KCs in co-IP experiments. Interestingly, RIPK3 also had a higher phosphorylation level in the ET group than in the NET group. Meanwhile, we noticed that the level of phosphorylated RIPK3 was decreased after knocking down the expression of TBK1. After increasing the expression of TBK1, the level of RIPK3 phosphorylation was also increased. Based on these data, we speculated that TBK1 could affect the phosphorylation level of RIPK3 in macrophages.

A switch in metabolism from oxidative phosphorylation to glycolysis occurs following activation of macrophages by LPS (McGettrick and O'Neill 2013; Tannahill et al. 2013). More importantly, this switch in the metabolic state directly impacts the inflammatory state of macrophages (McGettrick and O'Neill 2013). LPS administration also causes pronounced changes in macrophage metabolism and function (Nagy and Haschemi 2015). Among them, the metabolism of glutamine is profoundly affected during the macrophage activation process, and it is involved in the polarizing signals that upregulate the transcriptional programs required for macrophages to perform specialized functions (Cruzat et al. 2018). In response to cytokines and microbial products, macrophages express specialized and polarized functional properties; therefore, many refer to polarized macrophages as M1 and M2 cells (Gordon and Martinez 2010; Mantovani et al. 2005). M1 macrophages are part of the first line of defense of the innate immune system, which elicits rapid proinflammatory responses to infection, while M2 macrophages play a larger role in resolving inflammation and promoting tissue repair (Liu et al. 2017; Galván-Peña et al. 2014). Their metabolism is unsurprisingly a clear reflection of those functions (Mosser and Edwards 2008; Galván-Peña et al. 2014).

GLUD1, a metabolic enzyme that converts glutamine to a-KG, was identified in the RIPK3 complex in treated cells (Zhang et al. 2009). Our study showed that the GLUD1 and RIPK3 proteins interacted in ET models in vivo and in vitro, and the expression of GLUD1 and phosphorylated RIPK3 was higher in the ET group than in the NET group. Furthermore, the enzyme activity of GLUD1 and the serum and cell 
supernatant levels of a-KG were increased in the ET group compared with the NET group. The ratio of M1type macrophages in the ET group significantly decreased. Growing evidence has shown that a-KG generated from glutaminolysis promotes $\mathrm{M} 2$ activation and restricts $\mathrm{M} 1$ polarization, resulting in a crucial role for this metabolite in promoting tolerance induced by LPS (Cruzat et al. 2018; Liu et al. 2017). Interestingly, the regulatory effect of GLUD1 and a-KG on ET was reversed after eliminating macrophages with neutral liposomes: the percent survival of mice in the N-ET group was reduced by approximately $93 \%$ compared with that in the C-ET group; there were no significant differences in tissue injury or inflammatory cell infiltration between the N-NET group and the N-ET group; and the enzyme activity of GLUD1 was lower in the N-NET group than in the C-NET group, resulting in a decrease in a-KG. Taken together, GLUD1 and a-KG promote ET mainly by mediating the polarization of macrophages.

With the decrease in RIPK3, the expression of GLUD1 was significantly lower in the RIPK3-siRNA+ET group than that in the ET group. In addition, GLUD1 enzyme activity and a-KG levels were also decreased in the RIPK3-siRNA+ET group compared with the ET group, which resulted in an increase in the ratio of M1 macrophages in the RIPK3-siRNA+ET group. Correspondingly, when RIPK3 expression was increased, GLUD1 expression increased as well, which led to higher levels of a-KG.

\section{Conclusion}

Our study indicated that overexpression of RIPK3 can promote GLUD1 enzyme activity to increase the production of a-KG. Interestingly, we found that after knocking down the expression of TBK1, the expression of GLUD1 and enzyme activity of GLUD1 were decreased. Additionally, the level of a-KG was also decreased in the RIPK3-siRNA+ET group compared with the ET group, while the ratio of M1 macrophages was increased. In contrast, overexpression of TBK1 could mediate phosphorylation of RIPK3, thereby increasing the expression and enzyme activity of GLUD1 to achieve macrophage ET by enhancing the production of a-KG. Collectively, our study provides a novel pathway by which TBK1 participates in glutaminolysis by mediating the phosphorylation of RIPK3 to promote macrophage tolerance to endotoxin and unveils the potential for therapeutic targeting of the metabolic status of macrophages.

\section{Abbreviations}

\section{DMEM}

Dulbecco's modifed Eagle's medium

PVDF

Polyvinylidene difuoride

BSA

Albumin bovine

PBS

phosphate buffered saline

TBST 
Tris bufered saline with Tween-20

ELISA

Enzyme-linked immuno sorbent assay

ANOVA

Analysis of varianc

M-CSF

Colony stimulating factor.

\section{Declarations}

This study was supported by the National Natural Science Foundation of China (No. 81971880 and No. 81801963) and China Postdoctoral Science Foundation (2019M653352).

\section{Authors' contributions}

LP and LY put forward the research hypothesis and designed the experiments. LP, LY and ZY carried out the experimental procedures. LP carried out data analysis. WZ and JG were presided the study and provided financial support. This manuscript was prepared by LP, LY, ZY, WZ and JG. All authors contributed to the article and approved the submitted version.

\section{Funding}

This study was supported by the National Natural Science Foundation of China (No. 81971880 and No. 81801963) and China Postdoctoral Science Foundation (2019M653352).

\section{Availability of data and materials}

All data generated or analyzed during this study are included in this published article and its additional information files.

\section{Ethics approval and consent to participate}

Ethical consideration was approved by The Ethics Committee of the Second Affiliated Hospital of Chongqing Medical University (No. 42). All the animal experiments were performed in accordance with the ethical and legal guidelines.

\section{Competing interests}

The authors declare that they have no competing interests.

\section{Consent for publication}

None.

\section{Author details}


${ }^{1}$ Department of Hepatobiliary Surgery, The Second Affiliated Hospital of Chongqing Medical University, No.76 Linjiang Road, Yuzhong District, Chongqing, 400016, People's Republic of China. ${ }^{2}$ Department of Hepatobiliary Surgery, Chongqing University Three Gorges Hospital, No. 165 Xincheng Road, Wanzhou District, Chongqing, 404100, People's Republic of China.

\section{References}

1. Biswas SK, Bist P, Dhillon MK, et al. Role for MyD88-independent, TRIF pathway in lipid A/TLR4induced endotoxin tolerance. J Immunol. 2007;179:4083-92.

2. Biswas SK, Lopez-Collazo E. Endotoxin tolerance: new mechanisms, molecules and clinical significance. TRENDS IMMUNOL. 2009;30:475-87.

3. Chau TL, Gioia R, Gatot JS, et al. Are the IKKs and IKK-related kinases TBK1 and IKK-epsilon similarly activated? Trends Biochem Sci. 2008;33:171-80.

4. Chen S, Yang F, Cao W, et al. Quantitative Proteomics Reveals a Novel Role of the E3 Ubiquitin-Protein Ligase FANCL in the Activation of the Innate Immune Response through Regulation of TBK1 Phosphorylation during Peste des Petits Ruminants Virus Infection. J Proteome Res. 2021;20:411330.

5. Cruzat V, Macedo RM, Noel KK, Curi R, Newsholme P. Glutamine: Metabolism and Immune Function, Supplementation and Clinical Translation. Nutrients. 2018;10:1564.

6. Davies LC, Rice CM, Palmieri EM, et al. Peritoneal tissue-resident macrophages are metabolically poised to engage microbes using tissue-niche fuels. Nat Commun. 2017;8:2074.

7. Declercq W, Vanden BT, Vandenabeele P. RIP kinases at the crossroads of cell death and survival. Cell. 2009;138:229-32.

8. Del FC, García-Rio F, Gómez-Piña V, et al. Potent phagocytic activity with impaired antigen presentation identifying lipopolysaccharide-tolerant human monocytes: demonstration in isolated monocytes from cystic fibrosis patients. J Immunol. 2009;182:6494-507.

9. Dowling JP, Alsabbagh M, Del CC, et al. TRADD regulates perinatal development and adulthood survival in mice lacking RIPK1 and RIPK3. Nat Commun. 2019;10:705.

10. Galván-Peña S, O'Neill LA. Metabolic reprograming in macrophage polarization. Front Immunol. 2014;5:420.

11. Goossens V, Grooten J, Fiers W. The oxidative metabolism of glutamine. A modulator of reactive oxygen intermediate-mediated cytotoxicity of tumor necrosis factor in L929 fibrosarcoma cells. J Biol Chem. 1996;271:192-6.

12. Gordon S, Martinez FO. Alternative activation of macrophages: mechanism and functions. Immunity. 2010;32:593-604.

13. He $Y$, Lang $X$, Cheng $D$, et al. miR-30a-5p inhibits hypoxia/reoxygenation-induced oxidative stress and apoptosis in HK-2 renal tubular epithelial cells by targeting glutamate dehydrogenase 1 (GLUD1). Oncol Rep. 2020;44:1539-49. 
14. Ho PC, Tsui YC, Feng X, et al. NF-kB-mediated degradation of the coactivator RIP140 regulates inflammatory responses and contributes to endotoxin tolerance. Nat Immunol. 2012;13:379-86.

15. Jin J, Xiao Y, Chang JH, et al. The kinase TBK1 controls IgA class switching by negatively regulating noncanonical NF-KB signaling. Nat Immunol. 2012;13:1101-9.

16. Kang TB, Yang SH, Toth B, Kovalenko A, Wallach D. Caspase-8 blocks kinase RIPK3-mediated activation of the NLRP3 inflammasome. Immunity. 2013;38:27-40.

17. Liu D, Cao S, Zhou Y, Xiong Y. Recent advances in endotoxin tolerance. J Cell Biochem. 2019;120:5670.

18. Liu PS, Wang H, Li X, et al. a-ketoglutarate orchestrates macrophage activation through metabolic and epigenetic reprogramming. Nat Immunol. 2017;18:985-94.

19. Li PZ, Li JZ, Li M, et al. An efficient method to isolate and culture mouse Kupffer cells. Immunol Lett. 2014;158:52-6.

20. Mantovani A, Sica A, Locati M. Macrophage polarization comes of age. Immunity. 2005;23:344-6.

21. Mastorodemos V, Kotzamani D, Zaganas I, et al. Human GLUD1 and GLUD2 glutamate dehydrogenase localize to mitochondria and endoplasmic reticulum. Biochem Cell Biol. 2009;87:505-16.

22. McGettrick AF, O'Neill LA. How metabolism generates signals during innate immunity and inflammation. J Biol Chem. 2013;288:22893-8.

23. Moriwaki K, Chan FK. RIP3: a molecular switch for necrosis and inflammation. Genes Dev. 2013;27:1640-9.

24. Mosser DM, Edwards JP. Exploring the full spectrum of macrophage activation. Nat Rev Immunol. 2008;8:958-69.

25. Murray PJ, Wynn TA. Protective and pathogenic functions of macrophage subsets. Nat Rev Immunol. 2011;11:723-37.

26. Nagy C, Haschemi A. Time and Demand are Two Critical Dimensions of Immunometabolism: The Process of Macrophage Activation and the Pentose Phosphate Pathway. Front Immunol. 2015;6:164.

27. Nathan C, Ding A. Nonresolving inflammation. Cell. 2010;140:871-82.

28. Nelson VL, Nguyen $\mathrm{H}$, Garcia-Cañaveras JC, et al. PPARy is a nexus controlling alternative activation of macrophages via glutamine metabolism. Genes Dev. 2018;32:1035-44.

29. Netea MG, Balkwill F, Chonchol M, et al. A guiding map for inflammation. Nat Immunol. 2017;18:826-31.

30. Newton K. RIPK1 and RIPK3: critical regulators of inflammation and cell death. Trends Cell Biol. 2015;25:347-53.

31. Pasparakis M, Vandenabeele P. Necroptosis and its role in inflammation. Nature. 2015;517:311-20.

32. Perry AK, Chow EK, Goodnough JB, Yeh WC, Cheng G. Differential requirement for TANK-binding kinase-1 in type I interferon responses to toll-like receptor activation and viral infection. J Exp Med. 2004;199:1651-8. 
33. Petrasek J, Csak T, Szabo G. Toll-like receptors in liver disease. Adv Clin Chem. 2013;59:155-201.

34. Pomerantz JL, Baltimore D. NF-kappaB activation by a signaling complex containing TRAF2, TANK and TBK1, a novel IKK-related kinase. Embo J. 1999;18:6694-704.

35. Seeley JJ, Ghosh S. Molecular mechanisms of innate memory and tolerance to LPS. J Leukoc Biol. 2017;101:107-19.

36. Shin $\mathrm{CH}$, Choi DS. Essential Roles for the Non-Canonical IкB Kinases in Linking Inflammation to Cancer, Obesity, and Diabetes. Cells-Basel. 2019;8:178.

37. Speir M, Lawlor KE. RIP-roaring inflammation: RIPK1 and RIPK3 driven NLRP3 inflammasome activation and autoinflammatory disease. Semin Cell Dev Biol. 2021;109:114-24.

38. Su GL. Lipopolysaccharides in liver injury: molecular mechanisms of Kupffer cell activation. Am J Physiol Gastrointest Liver Physiol. 2002;283:G256-65.

39. Sun $L$, Wang $H$, Wang $Z$, et al. Mixed lineage kinase domain-like protein mediates necrosis signaling downstream of RIP3 kinase. Cell. 2012;148:213-7.

40. Sun X, Lee J, Navas T, et al. RIP3, a novel apoptosis-inducing kinase. J Biol Chem. 1999;274:168715.

41. Tannahill GM, Curtis AM, Adamik J, et al. Succinate is an inflammatory signal that induces IL-1 $\beta$ through HIF-1a. Nature. 2013;496:238-42.

42. Venet F, Monneret G. Advances in the understanding and treatment of sepsis-induced immunosuppression. Nat Rev Nephrol. 2018;14:121-37.

43. Wong WW, Vince JE, Lalaoui N, et al. clAPs and XIAP regulate myelopoiesis through cytokine production in an RIPK1- and RIPK3-dependent manner. Blood. 2014;123:2562-72.

44. Wynn TA, Chawla A, Pollard JW. Macrophage biology in development, homeostasis and disease. Nature. 2013;496:445-55.

45. Zhang DW, Shao J, Lin J, et al. RIP3, an energy metabolism regulator that switches TNF-induced cell death from apoptosis to necrosis. Science. 2009;325:332-6.

46. Zhao P, Wong KI, Sun X, et al. TBK1 at the Crossroads of Inflammation and Energy Homeostasis in Adipose Tissue. Cell. 2018;172:731-43.

\section{Figures}


Figure 1
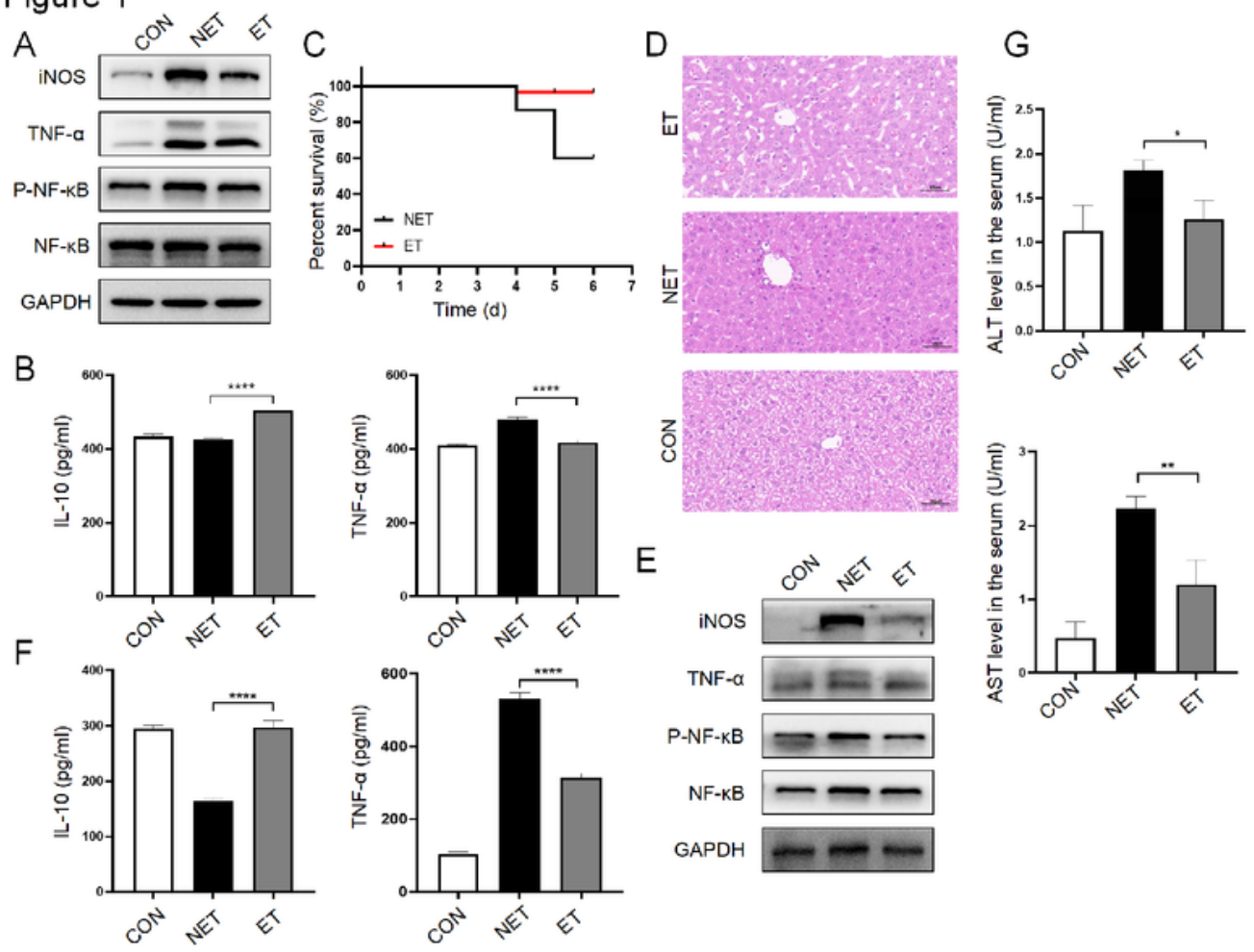

\section{Figure 1}

A low dose of LPS induces ET in vitro and in vivo. (A) Isolated KCs were randomized into the ET group, which was prestimulated with $10 \mathrm{ng} / \mathrm{mL}$ LPS for $24 \mathrm{~h}$, and the NET group. The two groups were subsequently stimulated with $1000 \mathrm{ng} / \mathrm{mL}$ LPS for $12 \mathrm{~h}$. All groups of KCs were sonicated, and the total protein supernatant was collected to compare the levels of TNF- $a$ and iNOS by western blotting. (B) IL-10 and TNF-a levels in KC supernatants were measured by ELISA. (C) Mouse survival was monitored every 
day after constructing nontolerant and tolerant models ( $n=15$ per group). (D) H\&E (400x) staining of mouse liver sections from the CON group, the NET group and the ET group was photographed to show the liver damage induced by LPS. (E) The protein expression levels of TNF-a and iNOS from tolerant and nontolerant mice were measured by western blotting $(n=3)$. ( $F)$ Serum IL-10 and TNF-a levels from tolerant and nontolerant mice were measured by ELISA $(n=3)$. (G) The levels of the ALT and AST in each group were detected $(n=3)$.

Data are presented as the means \pm SD of at least three independent experiments; ${ }^{*} p<0.05,{ }^{* \star} p<0.01$, $\star \star \star x p<0.001, * \star \star \star p<0.0001$. 
Figure 2

A

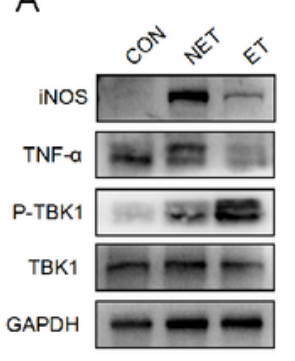

C

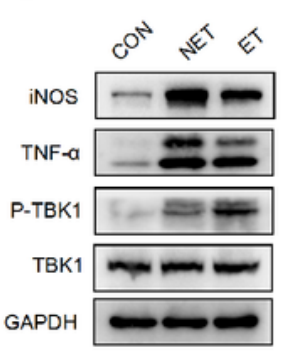

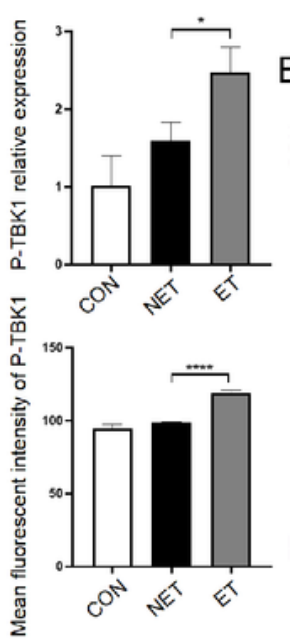

P-TBK1

DAPI

Merge
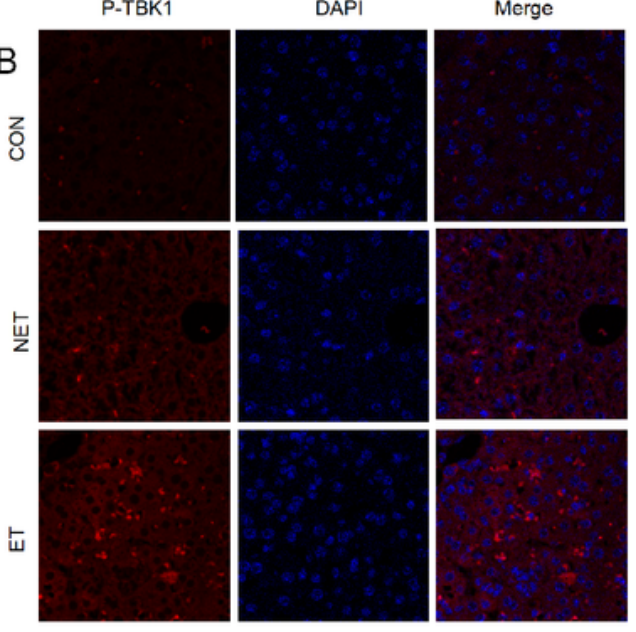

P-TBK1

DAPI
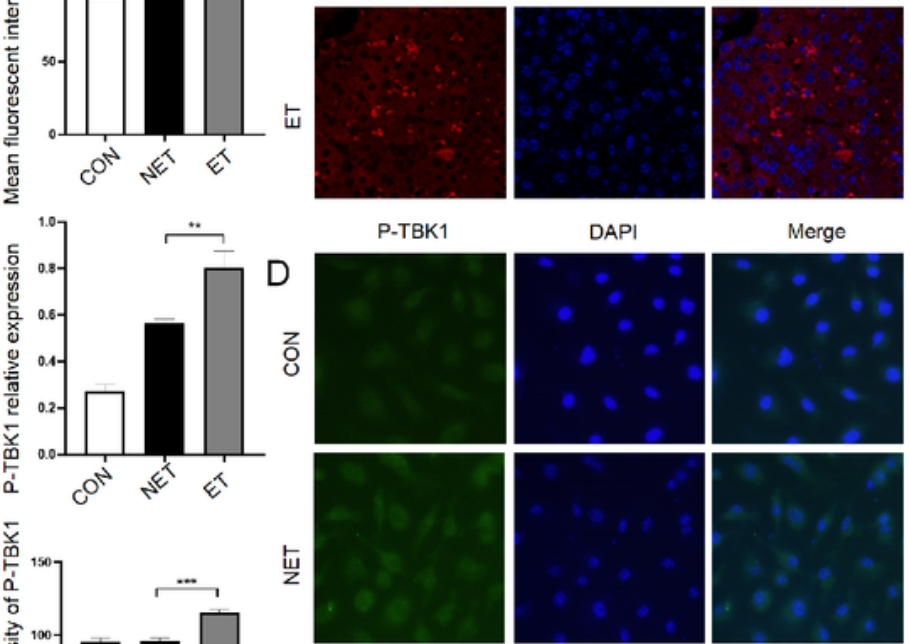

Merge
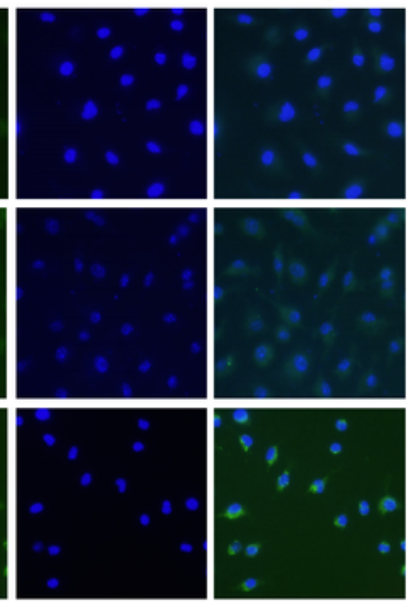

$$
\text { 党 }
$$
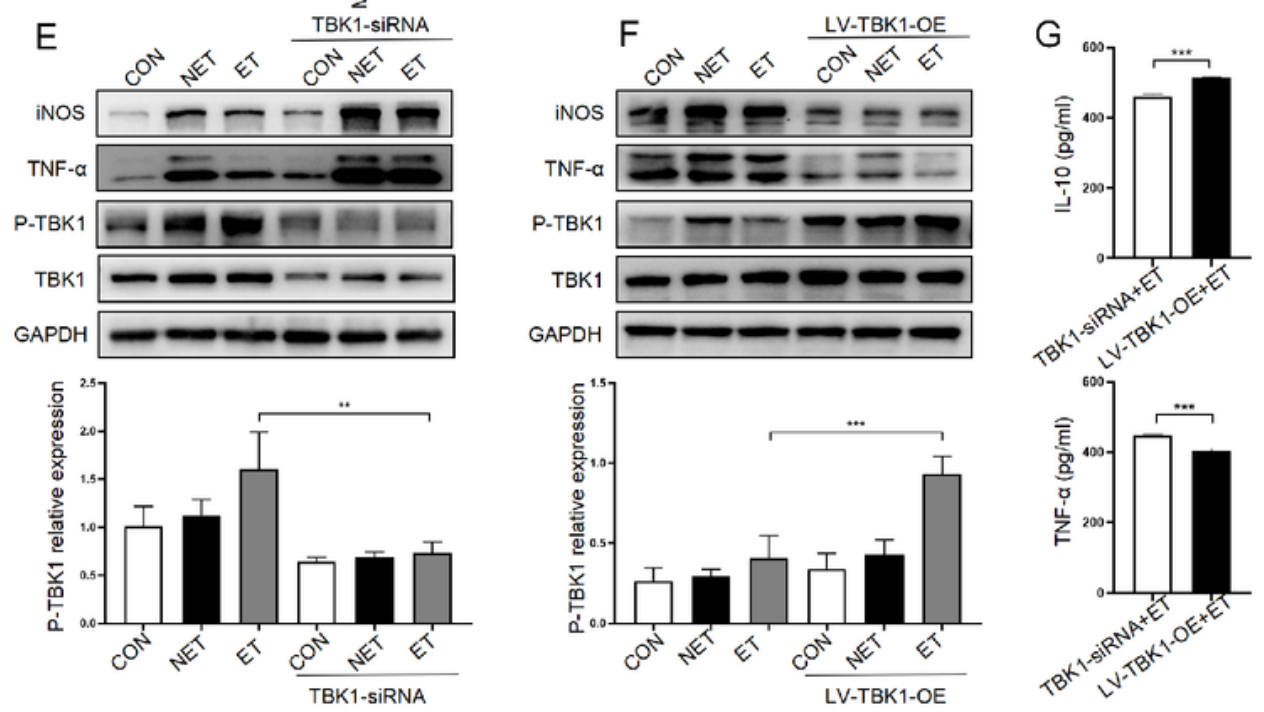

\section{Figure 2}

TBK1 can mediate inflammatory regulation in ET. (A) The protein expression levels of TBK1 and P-TBK1 in mice from the ET and NET groups were measured by western blotting $(n=3)$. (B) A higher phosphorylation level of TBK1 was found in the ET group by laser scanning confocal microscopy (LSCM) $(n=3)$. (C) The protein expression levels of TBK1 and P-TBK1 in KCs from the ET and NET groups were measured by western blotting ( $n=3)$. (D) The protein expression of P-TBK1 (green) and DAPI staining of 
the nucleus (blue) in KCs from the ET and NET groups were measured using immunofluorescence staining. (E) siRNA was used to knockdown the expression of TBK1 in RAW264.7 cells. The protein expression levels of IL-10, TNF-a, TBK1 and P-TBK1 were detected by western blotting $(n=3)$. (F) RAW264.7 cells were treated with shTBK1 lentiviruses for $72 \mathrm{~h}$. Protein expression of IL-10, TNF- $a$, TBK1 and P-TBK1 was measured by western blotting $(n=3)$. (G) Serum IL-10 and TNF-a levels from the siRNA+TBK1 ET group and LV-TBK1-OE group were measured by ELISA $(n=3)$. ${ }^{*} p<0.05, * * p<0.01$, *** $<$ $0.001, \star \star \star \star x<0.0001$. 
Figure 3

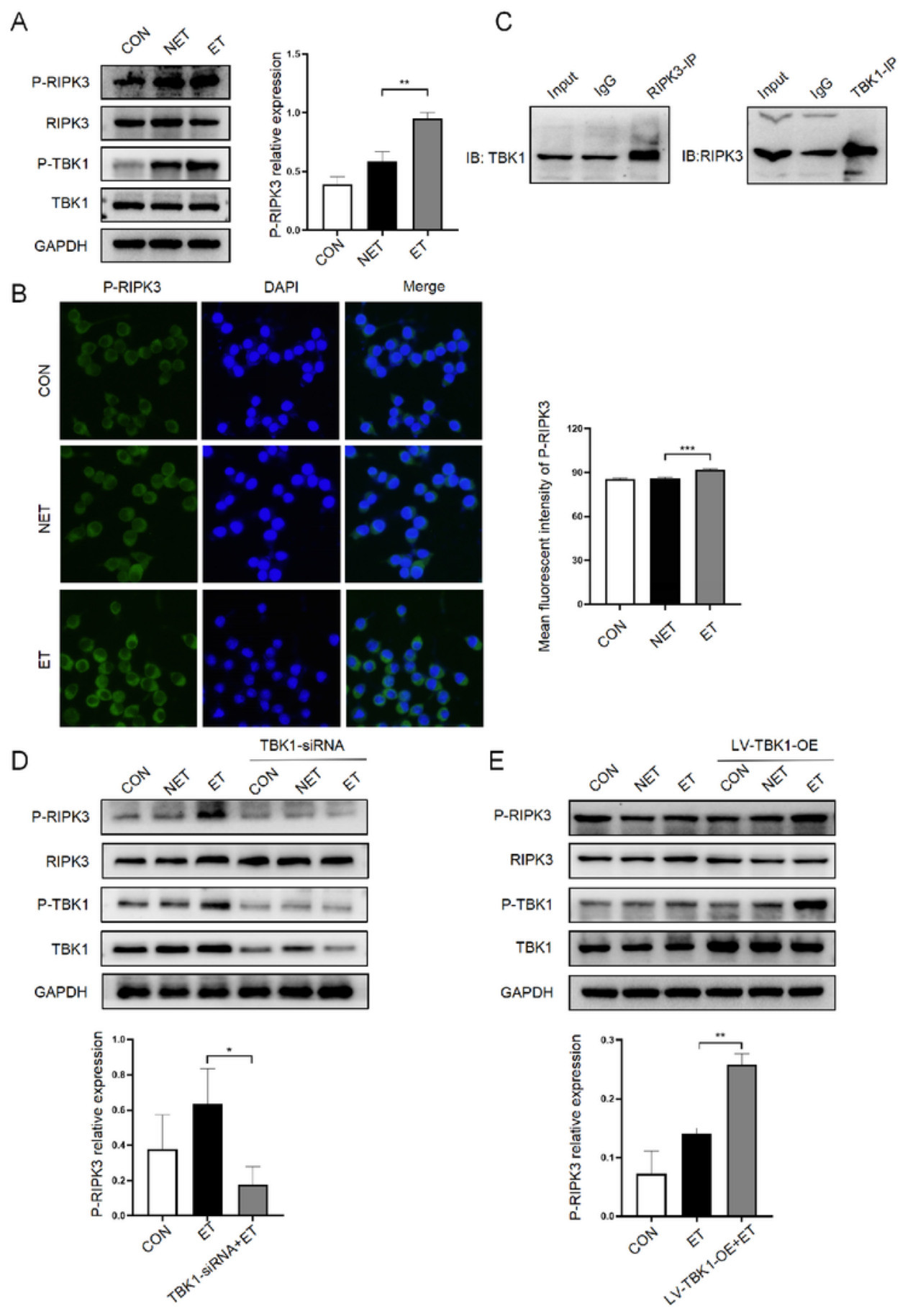

\section{Figure 3}

TBK1 can regulate the phosphorylation of RIPK3. (A) The protein expression of RIPK3 and P-RIPK3 in KCs from the ET and NET groups was measured by western blotting $(n=3)$. (B) The protein expression of PRIPK3 (green) and DAPI staining of the nucleus (blue) in KCs from the ET and NET groups were measured by immunofluorescence staining. (C) The interaction between RIPK3 and TBK1 in the ET group was detected by coimmunoprecipitation assays established in KCs. (D) Protein expression levels of IL-10, 
TNF- $a$, TBK1 and P-TBK1 were detected by western blotting $(n=3)$ after the expression of TBK1 was knocked down in RAW 264.7 cells. (E) RAW264.7 cells were treated with shRNA to increase the expression level of TBK1. Protein expression of RIPK3 and P-RIPK3 was measured by western blotting $(n=3) .{ }^{*}<$ $0.05,{ }^{* \star} \mathrm{p}<0.01,{ }^{\star * \star} \mathrm{p}<0.001$.

Figure 4
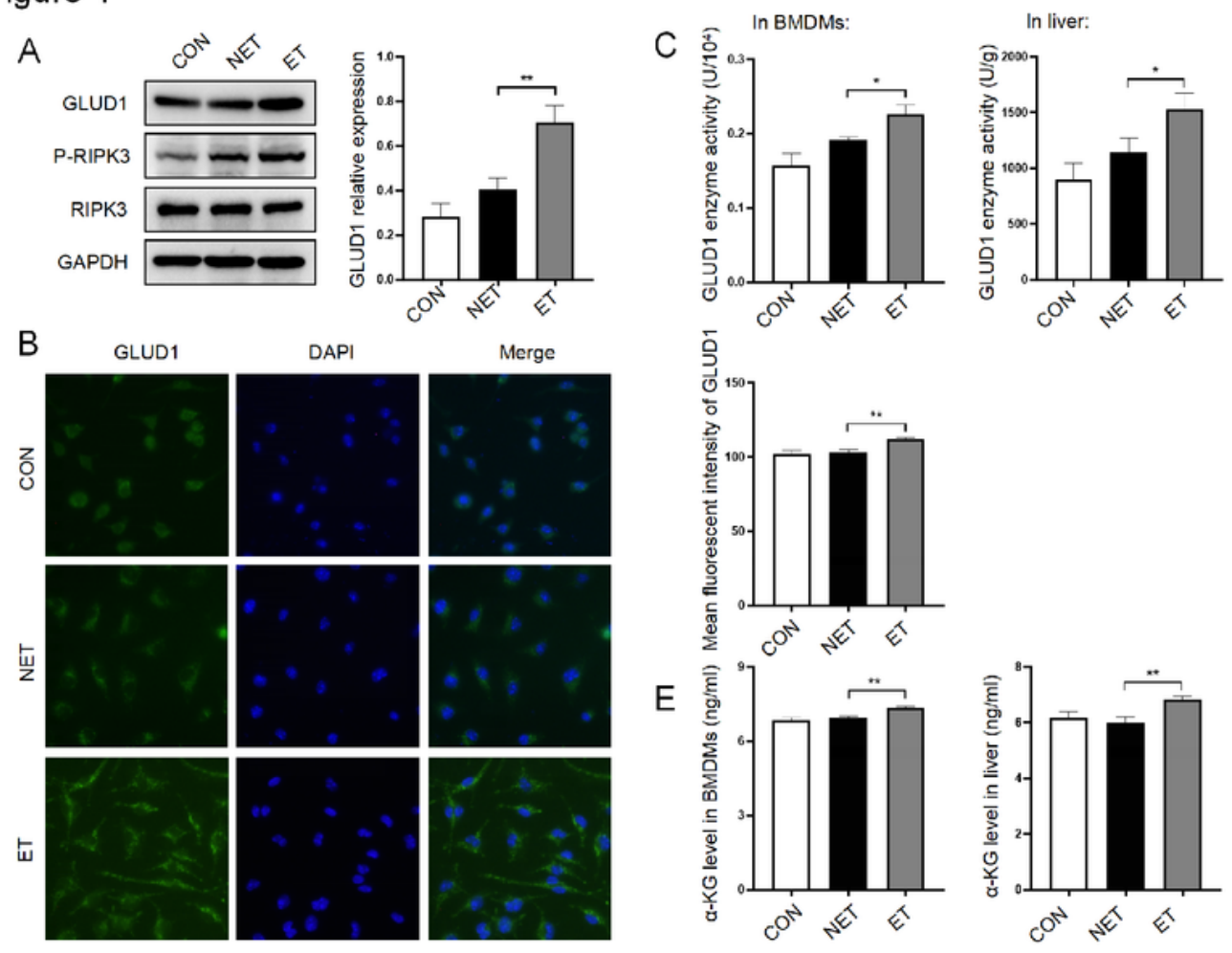

D
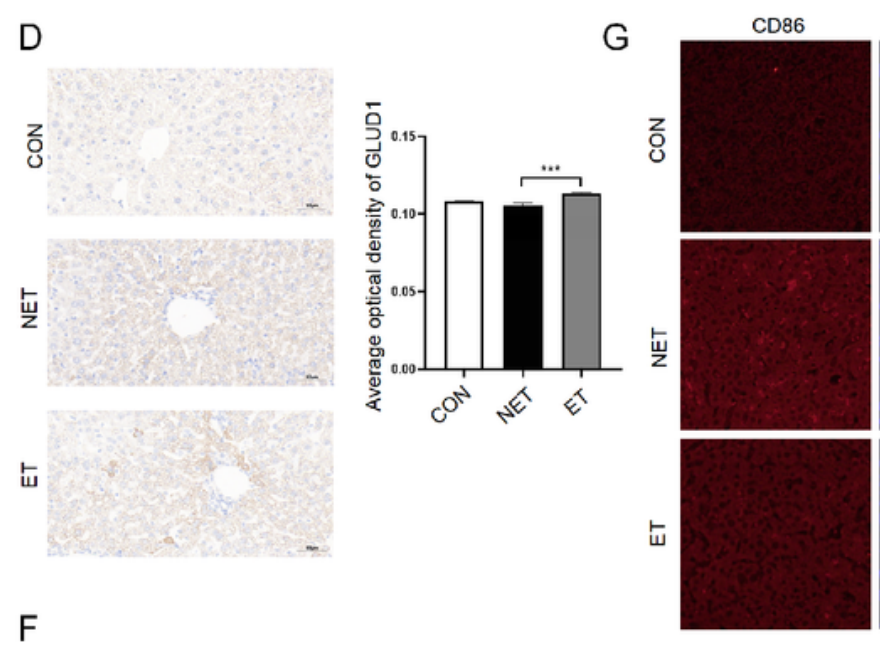

DAPI
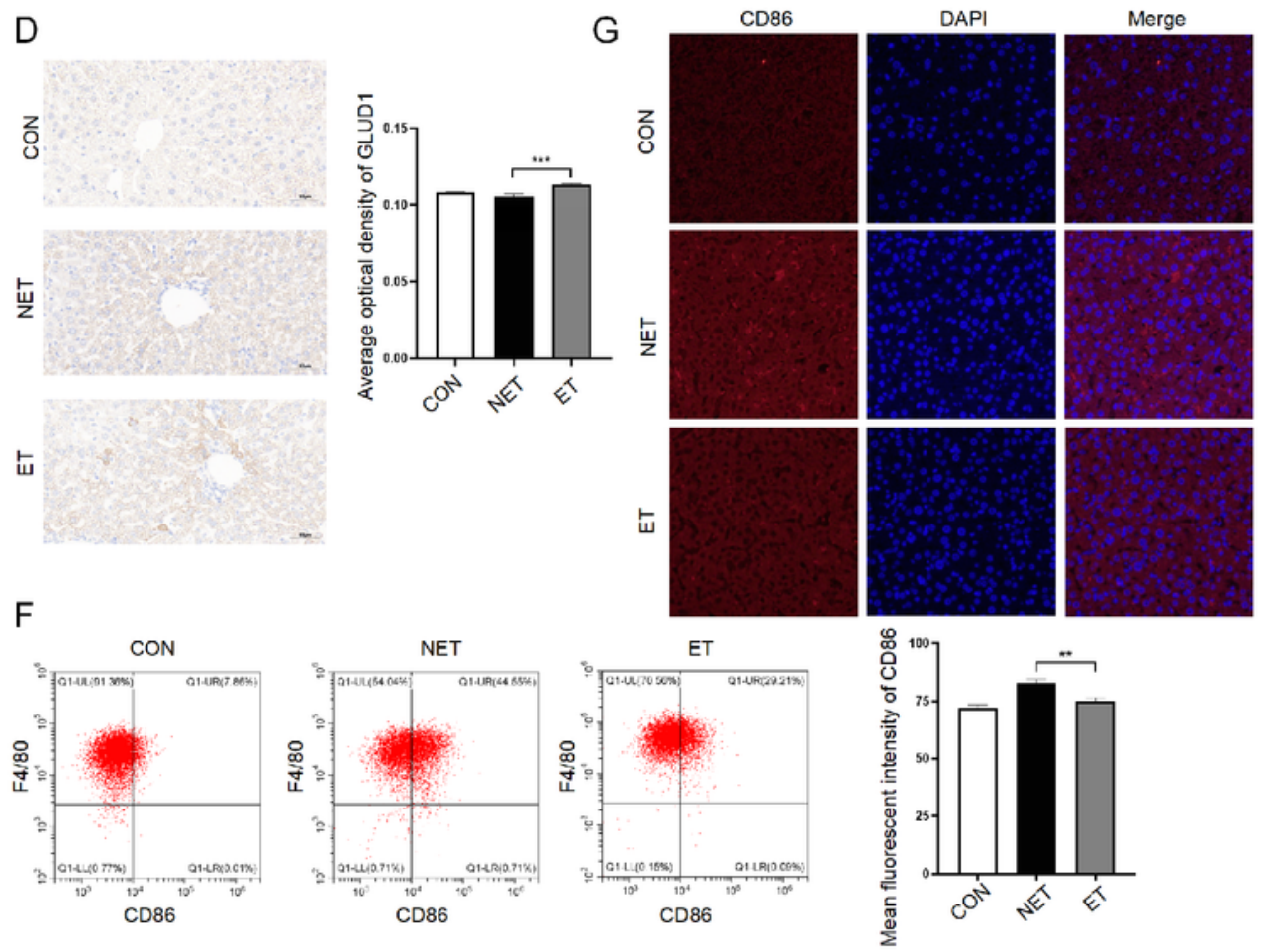

Figure 4 
Increasing the enzymatic activity of GLUD1 can induce ET in macrophages. (A) The protein expression of GLUD1 in mice from the ET and NET groups was detected by western blotting $(n=3)$. (B) The expression level of GLUD1 in BMDMs was measured using immunofluorescence staining. (C) The enzyme activities of GLUD1 in mice and in BMDMs. (D) Expression of GLUD1 in liver tissue was detected by immunohistochemical staining (400x) $(n=3)$. (E) The levels of $a-K G$ in serum and cell supernatants were assessed by ELISA $(n=3)$. $(F)$ The ratio of M1 macrophage phenotype-related surface markers CD86 and F4/80 was determined by flow cytometry for each group. (G) The expression of CD86 (red) and DAPI staining of the nucleus (blue) in livers from the ET and NET groups were measured by immunofluorescence staining. ${ }^{\star} p<0.05,{ }^{\star \star} p<0.01,{ }^{\star \star \star} p<0.001$. 
Figure 5

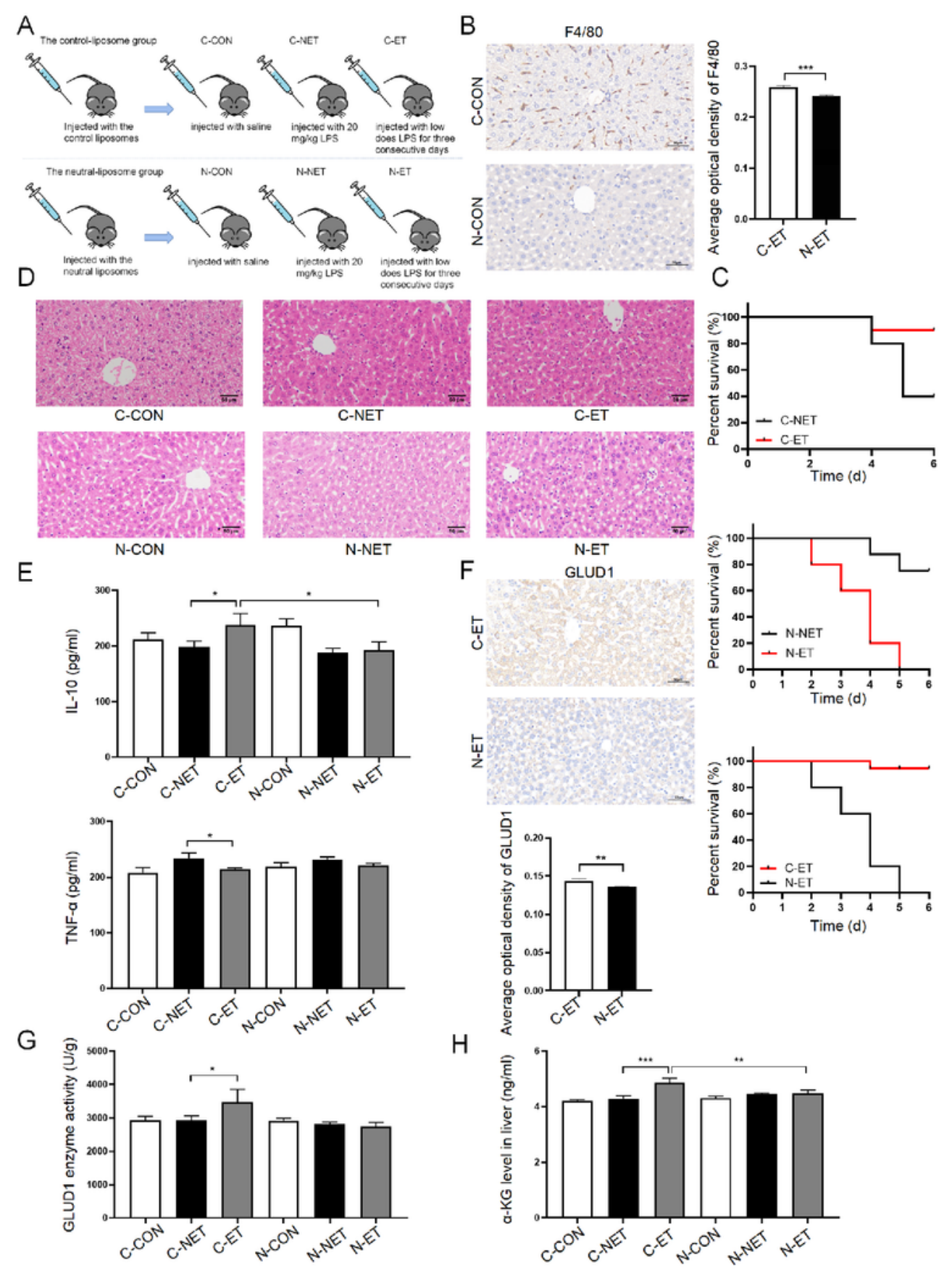

\section{Figure 5}

The regulatory effect of GLUD1 on ET was reversed after eliminating macrophages in mice. (A) The process of liposome injection in mice. (B) The expression of F4/80 in liver tissue was detected by immunohistochemical staining (400x) $(n=3)$. (C) Mouse survival was observed every day after constructing the control liposomes group (injected peritoneally with $200 \mu$ control liposomes) and the neutral liposome group (injected peritoneally with $200 \mu$ l neutral liposomes) ( $n=10$ per group). (D) H\&E 
(400x) staining of mouse liver sections was photographed to show the liver damage. (E) The serum levels of IL-10 and TNF-a were detected by ELISA $(n=3)$. ( $F)$ The expression of GLUD1 in liver tissue was detected by immunohistochemical staining (400x) $(n=3)$. (G) The enzyme activities of GLUD1 in livers from different groups $(n=3)$. $(H)$ Serum a-KG levels were measured by ELISA $(n=3) .{ }^{*} p<0.05,{ }^{* \star} p<0.01$.

Figure 6
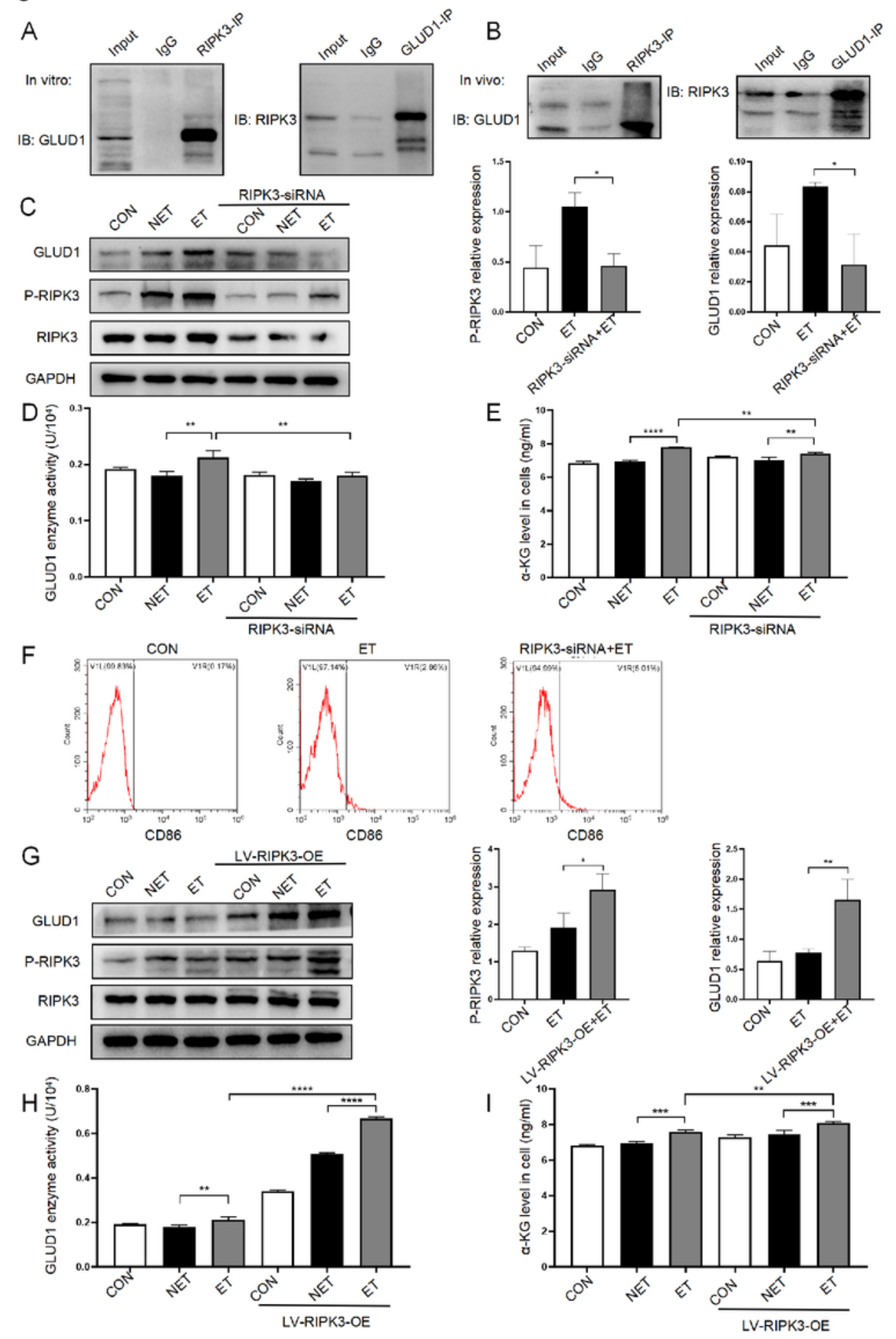

Figure 6 
RIPK3 induces ET by promoting the enzyme activity of GLUD1. (A and B) Interactions between RIPK3 and GLUD1 both in vivo and in vitro were assessed by coimmunoprecipitation assays. (C) The expression of RIPK3 was knocked down in RAW 264.7 cells by siRNA. The expression levels of P-RIPK3 and GLUD1 were detected by western blotting $(n=3)$. (D) The enzyme activity of GLUD1 in RAW 264.7 cells $(n=3)$. ( $E$ ) The level of a-KG in cell supernatnats was measured by ELISA ( $n=3)$. (F) The ratio of M1 macrophage phenotype-related surface markers (CD86) was determined by flow cytometry for each group. (G) Lentiviral particles were administered to increase RIPK3 expression in RAW264.7 cells. Protein expression of P-RIPK3 and GLUD1 was measured by western blotting $(n=3)$. (H) The enzyme activity of GLUD1 in RAW 264.7 cells after increasing the expression of RIPK3 $(n=3)$. (I) The level of a-KG in cell supernatants was measured by ELISA $(n=3) .{ }^{\star} p<0.05,{ }^{\star *} p<0.01,{ }^{* \star *} p<0.001,{ }^{\star \star \star *} p<0.0001$. 
Figure 7
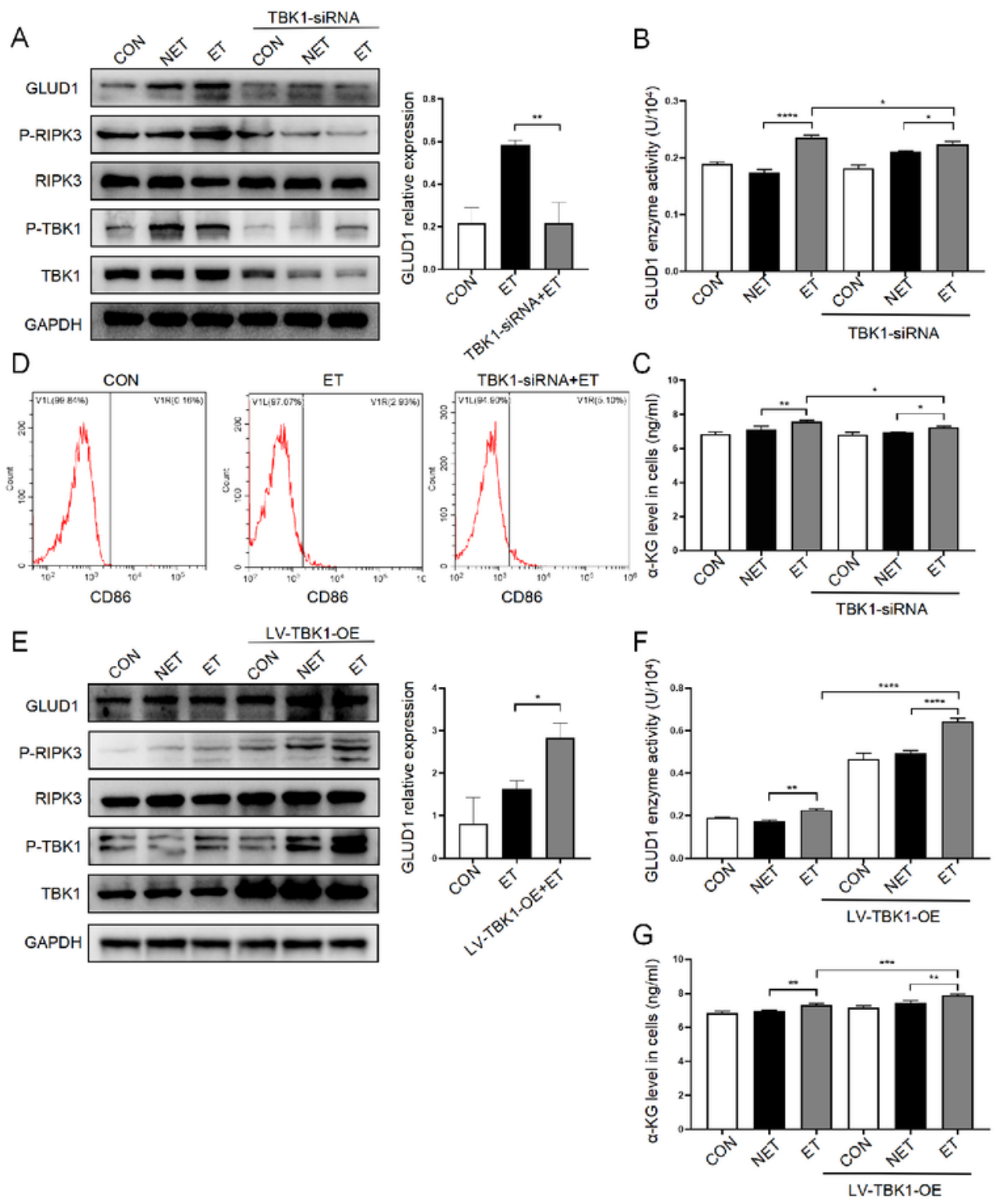

\section{Figure 7}

TBK1 participates in glutaminolysis by mediating the phosphorylation of RIPK3 to promote ET. (A) siRNA was used to knock down the expression of TBK1, and the protein expression of GLUD1 was measured $(n=3)$. (B) The enzyme activity of GLUD1 in RAW 264.7 cells after knocking down TBK1 $(n=3)$. (C) The level of a-KG in cell supernatants was measured by ELISA $(n=3)$. (D) The ratio of CD86 expression was determined by flow cytometry for each group. (E) Lentiviral particles were used to increase TBK1 
expression. Western blotting was performed to detect the expression of GLUD1 $(n=3)$. (F) The enzyme activities of GLUD1 in RAW 264.7 cells after increasing the expression of TBK1 $(n=3)$. (G) The level of aKG in cell supernatants was measured by ELISA $(n=3) .{ }^{\star} p<0.05,{ }^{* \star} p<0.01,{ }^{\star \star \star} p<0.001,{ }^{\star \star \star \star *} p<0.0001$.

\section{Figure 8}

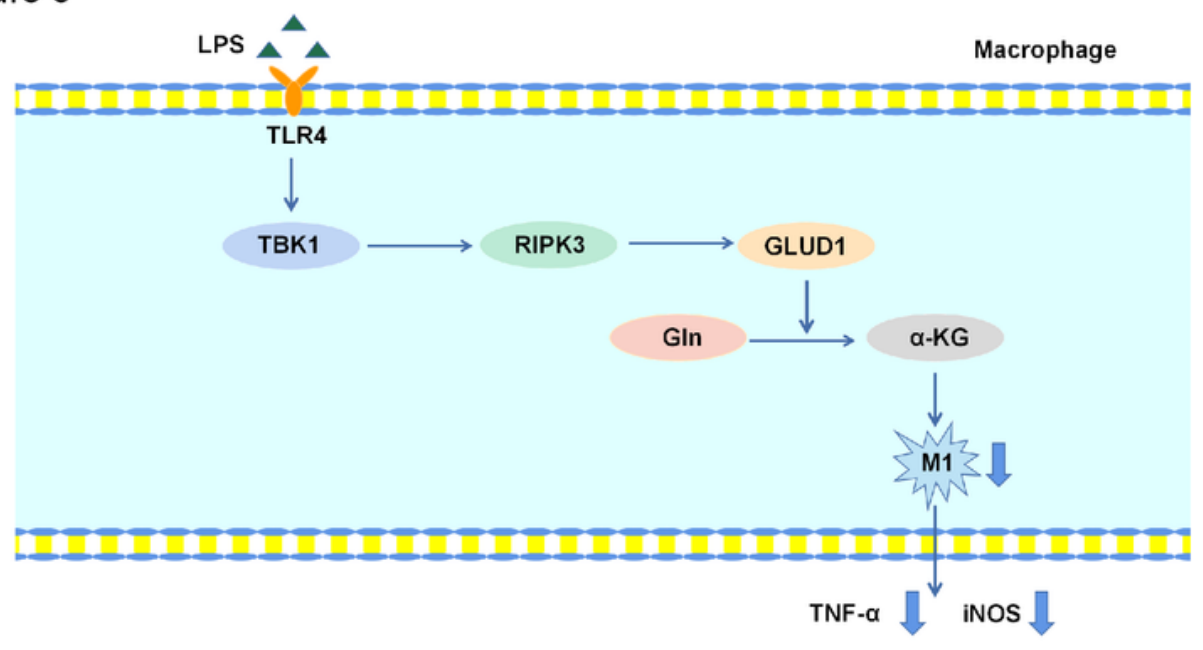

Figure 8 
The TBK1 protein directly interacts with the RIPK3 protein and mediates the phosphorylation of RIPK3 in macrophages. Then, activated RIPK3 directly improves the enzyme activity of GLUD1, which increases the production of $\mathrm{a}-\mathrm{KG}$ to restrict M1 polarization. 